\title{
Three-Dimensional Measurements of Micro- to Nanometer-Scale Features at and Below the Surface Using Scanning Electron Microcopy
}

\author{
Minhua Zhao***, Bin Ming*, Purushotham P. Kavuri*, and András E. Vladár* \\ * Mechanical Metrology Division, National Institute of Standards and Technology, Gaithersburg, \\ MD 20899 \\ ** Institute for Research in Electronics and Applied Physics, University of Maryland, College Park, \\ MD 20742
}

Three-dimensional (3D) imaging with a scanning electron microscope (SEM) has been the object of previous studies [1-3], because lack of quantitative information on third dimension is one of the limitations of SEM techniques. Although these studies allow for good qualitative and limited quantitative 3D SEM measurements on the surface, the size of sample features is limited to tens of micrometers or larger. So far there are few studies on SEM 3D measurement of micro- and nanometer -scale features at or below the surface. In this study, 3D measurement of micro- and nanometer-scale features at and below the surface were conducted based on stereo-pair images acquired by a high-resolution field emission SEM.

In Fig. 1, a NIST RM 8820 reference sample with $2 \mu \mathrm{m}$ pitch was chosen as a micrometer-scale feature at the surface. While the error of overall measured height step is within $20 \%$ of nominal value $(97.3 \mathrm{~nm}$ with a standard deviation of $1.6 \mathrm{~nm})$, there is a significant measurement error near the step edge, which can be reduced using our previous Monte-Carlo electron transport modelling $[4]$.

In Fig. 2, a single wall carbon nanotube (SWCNT) polyimide composite film [5] was used as a nanometer-scale feature for measurements below the surface. Remarkable 3D reconstruction of SWCNTs embedded in the polymer composite has been demonstrated. The accuracy of the 3D measurements of embedded features is not a trivial issue, and for solution, suitable metrology methods are now under development. This work reveals the potentials of SEM 3D imaging of nanometer-scale features below the surface [6-7], which will benefit a range of applications in nanotechnology.

\section{Reference:}

[1] F. Marinello et al, Measurement Science and Technology, 19(2008)065705.

[2] W. Drzazga et al, Measurement Science and Technology, 17(2006)28.

[3] M. Marko, Microscopy and Microanalysis, 11(2005) 624.

[4] J.S. Villarrubia et al, Surf. Interface Anal., 37(2005)951.

[5] M.H. Zhao et al, Nanotechnology, 21(2010) 225702.

[6] M.H. Zhao et al, Nanotech 2010, paper \# 884, Anaheim, CA, 2010.

[7] M.H. Zhao et al, manuscript in preparation.

[8] Dr. Zhao was supported by the NIST-ARRA senior fellowship in measurment science and technology. The CNT-polyimide composite films are provided by Dr. Cheol Park from National Institute of Aerospace. The help of Dr. Roger Alvis of FEI company is gratefully acknowledged. 


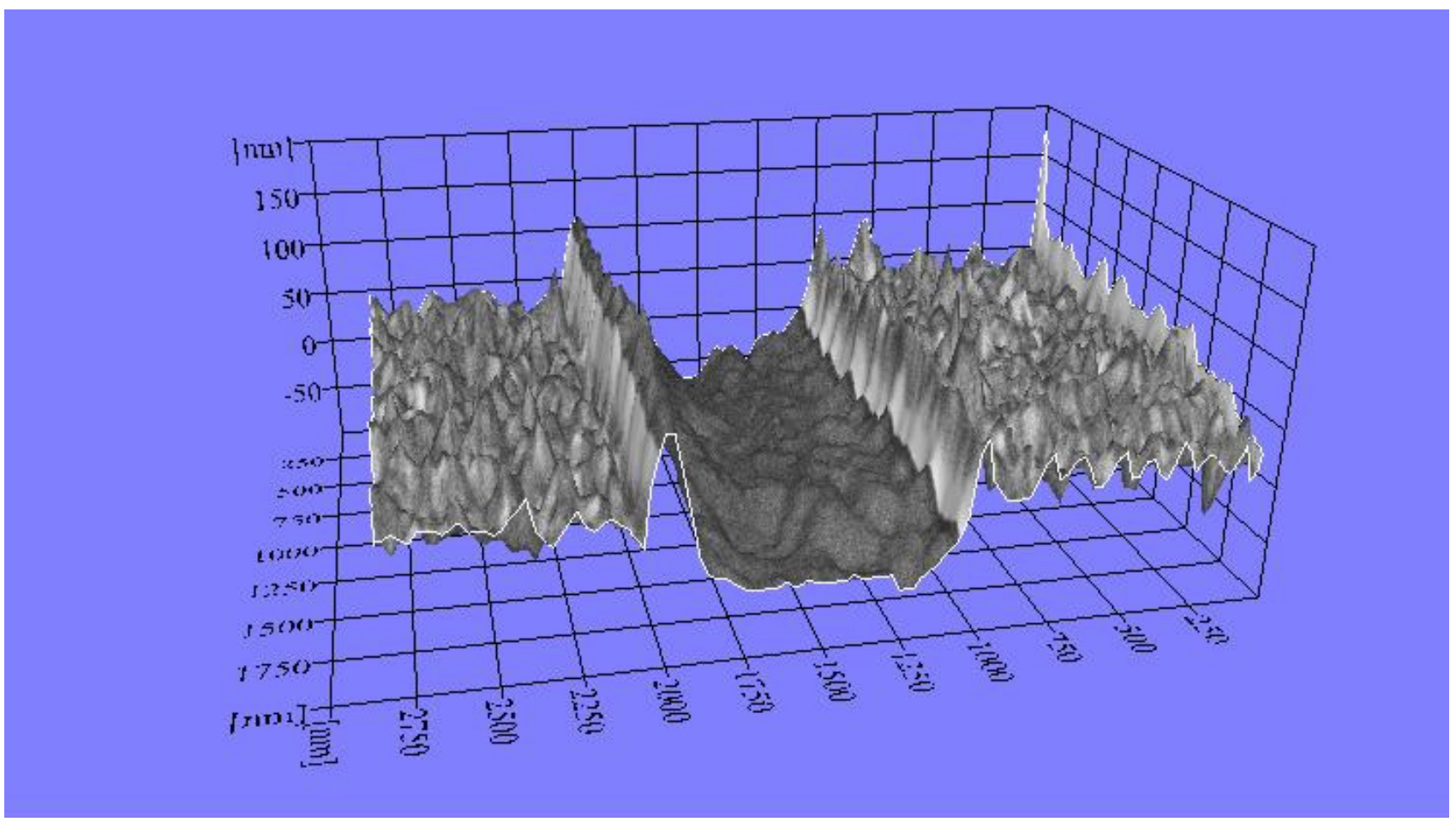

FIG. 1. SEM 3D measurement of NIST RM 8820 reference sample with $2 \mu \mathrm{m}$ pitch and step height of $97 \mathrm{~nm}$.

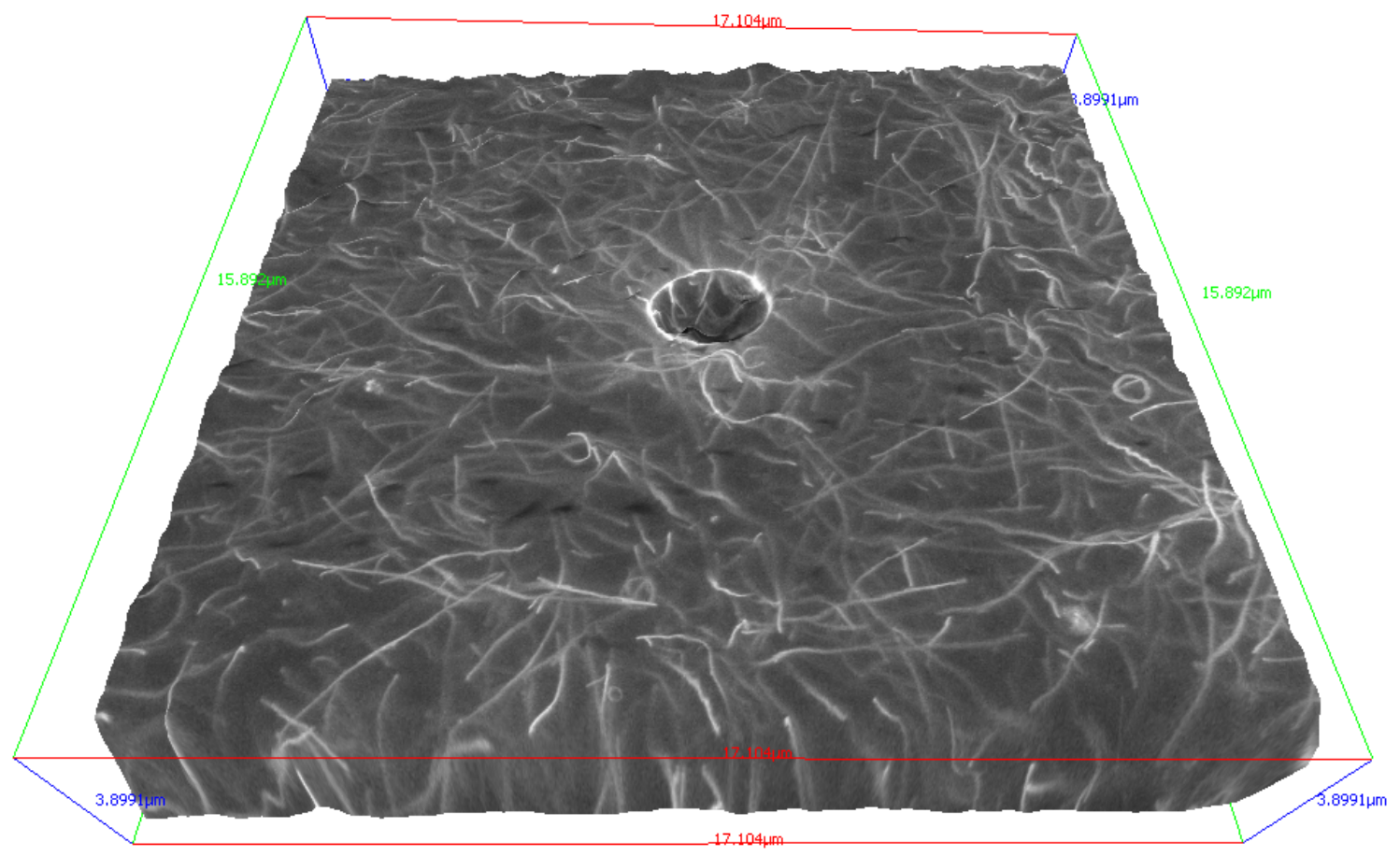

FIG. 2. SEM 3D reconstruction of $0.5 \%$ SWCNT-polyimide composite sample. 